\title{
Caractérisation des cas de syphilis infectieuse féminine en Colombie-Britannique afin de déterminer les possibilités d'optimisation des soins
}

\author{
Kaylie Willemsma ${ }^{1 *}$, Lindsay Barton², Rochelle Stimpson², Irene Pickell², Venessa Ryan², \\ Amanda Yu², Ann Pederson 1,3, Gina Ogilvie ${ }^{1,2,3}$, Troy Grennan 1,2, Jason Wong ${ }^{1,2}$
}

\section{Résumé}

Introduction : Le taux de syphilis infectieuses continue d'augmenter chez les femmes en Colombie-Britannique et au Canada, ce qui soulève des préoccupations quant à l'augmentation de l'incidence de la syphilis congénitale. Nous avons caractérisé les cas de syphilis chez les femmes de la Colombie-Britannique afin de déterminer les possibilités de prévenir la syphilis et d'optimiser ses soins.

Méthodes : Tous les cas de syphilis infectieuse diagnostiqués en Colombie-Britannique entre le 13 mars 2018 et le 31 décembre 2020 et s'identifiant comme femme ont été examinés. Des données démographiques, des facteurs de risque et des conditions concurrentes ont été recueillis auprès d'un système de surveillance provincial. Des analyses de sous-groupes comparant des cas avec et sans instabilité du logement, consommation de substances, maladie mentale et une infection sexuellement transmissible récente (ITS) ont été effectuées afin de comprendre les différences entre ces sous-groupes. Les associations statistiques ont été calculées au moyen de tests khi carré ou de tests $t$.

Résultats : Au cours de cette période, 226 cas de syphilis infectieuse féminine ont été déclarés en Colombie-Britannique : 38 (16,8 \%) en 2018; 74 (32,7\%) en 2019; et 114 (50,4 \%) en 2020. L'âge moyen était de 32 ans (intervalle de 15 à 75 ans). Parmi les personnes qui ont déclaré des conditions concomitantes, la plupart des cas ont connu une instabilité du logement $(71,1 \%)$, une consommation de substances $(68,2 \%)$ et une maladie mentale $(83,9 \%)$, alors que $42,9 \%$ avaient une ITS récente. Les cas qui ont déclaré une instabilité du logement ou une consommation de substances étaient beaucoup plus susceptibles d'avoir récemment eu une ITS, avoir des antécédents de vie dans la rue, de rapports sexuels transactionnels, une maladie mentale et une aide au revenu (tous $p<0,01$ ).

Conclusion : Nos résultats soulignent l'importance de favoriser un environnement propice aux soins de la syphilis. Des services simultanés pour soutenir les personnes atteintes de syphilis ainsi que l'instabilité du logement, la consommation de substances et la maladie mentale pourraient aider à prévenir la syphilis et à améliorer le bien-être.
Cette oeuvre est mise à la disposition selon les termes de la licence internationale Creative Commons Attribution 4.0

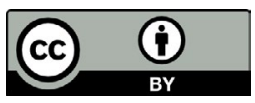

Affiliations

${ }^{1}$ Faculté de médecine, Université de la Colombie-Britannique, Vancouver, BC

${ }^{2}$ Centre de contrôle des maladies de la Colombie-Britannique, Vancouver, BC

${ }^{3}$ BC Women's Hospital, Vancouver, BC

*Correspondance : kaywill@student.ubc.ca

Citation proposée : Willemsma K, Barton L, Stimpson R, Pickell I, Ryan V, Yu A, Pederson A, Ogilvie G, Grennan T, Wong J. Caractérisation des cas de syphilis infectieuse féminine en Colombie-Britannique afin de déterminer les possibilités d'optimisation des soins. Relevé des maladies transmissibles au Canada 2022;48(2/3):76-84. https://doi.org/10.14745/ccdr.v48i23a03f

Mots-clés : syphilis infectieuse féminine, infections transmissibles sexuellement, syndémies, déterminants sociaux de la santé, consommation de substances, instabilité résidentielle, Canada 


\section{Introduction}

Les taux de syphilis infectieuse ont augmenté en

Colombie-Britannique et partout au Canada. Bien que la syphilis infectieuse affecte de façon disproportionnée les hommes gais, bisexuels ou ayant des relations sexuelles avec d'autres hommes (gbHARSAH), un nombre croissant de cas sont déclarés parmi les populations féminines et hétérosexuelles, ce qui soulève des préoccupations quant à l'incidence accrue de la syphilis congénitale. De 2016 à 2020, les taux de syphilis infectieuse féminine ont augmenté de $740 \%$ au Canada (1). À I'heure actuelle, un cas sur trois de syphilis infectieuse au Canada concerne des femmes (1). Parallèlement, il y a eu une augmentation du nombre de cas de syphilis congénitale à l'échelle nationale, de 4 cas en 2016 à 50 cas en 2020 (1).

En Colombie-Britannique, le taux de syphilis infectieuses a augmenté de $138 \%$ chez les femmes de 2018 à 2020 (2). Le ratio hommes-femmes des cas de syphilis infectieuse en Colombie-Britannique a diminué, passant de 15,9 en 2018 à 6,7 en 2020 (2). II convient de noter que le nombre de cas de syphilis infectieuse déclarés en 2020 pourrait être une sous-estimation en raison de l'accès réduit aux services de santé et des préoccupations liées à la maladie à coronavirus 2019 (COVID-19) (3). La Colombie-Britannique a déclaré trois cas de syphilis congénitale précoce en 2019; les premiers cas déclarés en six ans (4). En réponse, le Centre de contrôle des maladies de la Colombie-Britannique (BCCDC) a cherché à mettre à jour son plan d'action provincial en matière de syphilis (5), en mettant davantage l'accent sur la prévention de la syphilis et des complications connexes chez les femmes.

Pour éclairer la mise à jour du Plan d'action contre la syphilis, le BCCDC a mené des consultations avec les intervenants en fonction des piliers décrits dans le Cadre d'action pancanadien sur les infections transmissibles sexuellement et par le sang $(6,7)$. Les consultations ont mis l'accent sur la nécessité d'une approche syndémique pour lutter contre la syphilis $(7,8)$. La théorie des syndémies suppose que les problèmes de santé ont tendance à coexister, à être synergiques et à exacerber le fardeau de la santé chez les populations marginalisées (8). En particulier, les consultations ont mis en lumière la nécessité de s'attaquer à des conditions concurrentes, y compris l'instabilité du logement, la consommation de substances et la maladie mentale, ainsi que des facteurs systémiques tels que la relation avec un fournisseur de soins de santé dans le cadre de la réponse provinciale à la syphilis $(6,7)$. Afin de mieux comprendre la prévalence de ces conditions concomitantes et de ces facteurs de risque et de déterminer les possibilités d'optimiser les soins pour les femmes touchées par la syphilis, nous avons examiné tous les cas de syphilis infectieuse chez les femmes déclarées en Colombie-Britannique.

\section{Méthodes}

\section{Contexte}

Les cliniciens du BCCDC ont effectué ou coordonné la gestion des cas et des partenariats avec des fournisseurs de tests pour tous les cas de syphilis infectieuse diagnostiqués en Colombie-Britannique afin d'assurer des tests, un traitement, et un suivi approprié. Depuis le 13 mars 2018, tous les renseignements sur les cas et les partenaires ont été saisis dans les systèmes provinciaux de dossiers médicaux électroniques (DME) du profil Intrahealth.

\section{Critères d'inclusion}

Cette étude descriptive comprenait un examen rétrospectif de tous les cas féminins de syphilis infectieuse (i.e. stade primaire, secondaire ou latent précoce) diagnostiqués en Colombie-Britannique du 13 mars 2018 au 31 décembre 2020. Les définitions de cas pour les stades infectieux de la syphilis sont disponibles sur le site Web du BCCDC (9).

\section{Source de données}

Les cas de syphilis infectieuse féminine ont été identifiés par le système de DME du profil Intrahealth. Des détails sur les cas, y compris les données démographiques, les facteurs de risque et les conditions concurrentes, ont été recueillis par l'entremise du système de DME et du système CareConnect. CareConnect est un visualiseur intégré de l'information clinique provenant de l'ensemble de la Colombie-Britannique, y compris les rencontres cliniques et les renseignements de laboratoire (10).

\section{Variables d'intérêt}

La géographie était fondée sur les limites géographiques des autorités sanitaires de la Colombie-Britannique (11). L'urbanité était définie comme étant la vie dans une région métropolitaine comptant plus de 500000 habitants.

L'établissement du dépistage de la syphilis a été déterminé à l'aide de l'adresse du médecin ordonnateur et classé comme 1) les milieux communautaires (y compris la pratique de première ligne, les cliniques spécialisées et les cliniques sans rendez-vous), 2) les milieux hospitaliers (y compris les services hospitaliers, les services ambulatoires et les services d'urgence) ou 3) les milieux de sensibilisation (y compris les cliniques axées sur les soins aux personnes atteintes du virus de l'immunodéficience humaine $[\mathrm{VIH}]$, de la consommation de substances ou de la maladie mentale).

Les notes explicatives ont été examinées pour comprendre la raison du dépistage qui a abouti au diagnostic de syphilis. La raison des tests a été classée comme 1) contact avec une infection transmise sexuellement (ITS), 2) examen médical d'immigration, 3) incident, 4) prénatal ou à l'accouchement, 5) dépistage de routine ou 6) symptomatique. 
Une ITS récente a été définie comme un diagnostic de chlamydia, de gonorrhée ou de syphilis au cours des cinq années précédant la date du diagnostic de la syphilis.

Les conditions concourantes (e.g. instabilité du logement, la vie dans la rue, consommation de substances et maladie mentale) étaient fondées sur la documentation de l'état dans les notes cliniques ou comme diagnostic dans les DME avec un code international de classification des maladies. Par souci de simplicité, la maladie mentale a été catégorisée comme oui/ non. Les types de substances utilisées et l'état du logement au moment du diagnostic ont également été recueillis lorsqu'ils ont été documentés.

Le nombre de partenaires incluait tous ceux déclarés pendant la période infectieuse de syphilis. La notification aux partenaires était jugée complète si les cliniciens de la syphilis étaient en mesure d'informer tous les partenaires ou de faire toutes les tentatives raisonnables pour le faire, ou si le cas indiquait qu'ils en informeraient eux-mêmes leurs partenaires. Le lien avec un prestataire de soins primaires était fondé sur des preuves de visites et de tests répétés du même fournisseur.

Les codes postaux ont été utilisés pour estimer la situation socioéconomique à l'aide de l'Indice canadien de défavorisation multiple de Statistique Canada dans quatre domaines:

1) la composition ethnoculturelle; 2) la vulnérabilité de la situation; 3) la dépendance économique; et 4) I'instabilité résidentielle (12). Si le client n'avait pas d'adresse fixe ou de code postal inconnu, le code postal du médecin ordonnateur a été utilisé à la place.

\section{Analyses statistiques}

Des analyses descriptives ont été effectuées sur la cohorte complète de cas de syphilis infectieuse féminine, et une sous-analyse plus poussée a été effectuée sur les cas maternels. Des tests khi carré ou tests t ont été effectués sur quatre sous-groupes : 1) I'instabilité du logement; 2) la consommation de substances; 3 ) la maladie mentale; et 4) une ITS récente afin d'explorer les associations entre des conditions concomitantes. Ces sous-groupes ont été choisis en raison de leur association connue avec la syphilis et de leur potentiel de prévention de la syphilis, tel qu'il est décrit dans le Cadre pancanadien de lutte contre la syphilis et dans le Plan d'action de la Colombie-Britannique en matière de syphilis (5-7). La signification statistique a été définie comme $p<0,01$.

\section{Éthique}

Cette étude a été entreprise pour appuyer les efforts de surveillance et de prévention de la syphilis qui relèvent du mandat du BCCDC en matière de santé publique; par conséquent, l'approbation éthique n'était pas requise.

\section{Résultats}

En Colombie-Britannique, il y a eu 226 cas déclarés de syphilis infectieuse chez les femmes entre le 13 mars 2018 et le 31 décembre 2020. Le nombre de cas est passé de 38 (16,8 \%) en 2018 à 74 (32,7\%) en 2019 et à $114(50,4 \%)$ en 2020. Le nombre moyen de cas par mois était de $4,0,6,2$ et 9,5 pour 2018, 2019 et 2020, respectivement. L'âge moyen au moment du diagnostic était de 32 ans (intervalle de 15 à 75 ans). Les cas ont été répartis dans l'ensemble de la Colombie-Britannique, plus de la moitié d'entre eux résidant dans les deux autorités sanitaires les plus peuplées de la Colombie-Britannique (i.e. Vancouver Coastal Health et Fraser Health Authority). Plus des deux tiers ont été diagnostiqués au début du stade latent de l'infection (tableau 1).

\section{Tableau 1 : Statistiques descriptives des cas de syphilis infectieuse chez les femmes}

\begin{tabular}{|l|r|r|}
\hline \multicolumn{1}{|c|}{ Variables } & \multicolumn{1}{c|}{$\mathbf{n}$} & $\%$ \\
\hline Age (années) $(\mathrm{n}=226)$ & 32,5 & s.o. \\
\hline Moyenne & 30,9 & s.o. \\
\hline Médiane & 15,5 & s.o. \\
\hline Min. & 75,4 & s.o. \\
\hline Max. & &
\end{tabular}

Catégories d'âge $(n=226)$

\begin{tabular}{|c|c|c|}
\hline Moins de 20 ans & 18 & $8,0 \%$ \\
\hline 20 à 30 ans & 81 & $35,8 \%$ \\
\hline 30 à 40 ans & 92 & $40,7 \%$ \\
\hline 40 à 50 ans & 16 & $7,1 \%$ \\
\hline Plus de 50 ans & 19 & $8,4 \%$ \\
\hline \multicolumn{3}{|l|}{ Année - total $(n=226)$} \\
\hline $2018^{a}$ & 38 & $16,8 \%$ \\
\hline 2019 & 74 & $32,7 \%$ \\
\hline 2020 & 114 & $50,4 \%$ \\
\hline \multicolumn{3}{|c|}{ Année - nombre moyen de cas par mois $(n=226)$} \\
\hline $2018^{a}$ & 4.0 & s.o. \\
\hline 2019 & 6.2 & s.o. \\
\hline 2020 & 9.5 & s.o. \\
\hline \multicolumn{3}{|c|}{ Autorités sanitaires $(n=223$ ) } \\
\hline Nord & 9 & $4,0 \%$ \\
\hline Intérieur & 24 & $10,8 \%$ \\
\hline Île de Vancouver & 59 & $26,5 \%$ \\
\hline Fraser & 61 & $27,4 \%$ \\
\hline Vancouver Coastal & 70 & $31,4 \%$ \\
\hline \multicolumn{3}{|l|}{ Urbanité $(n=213)$} \\
\hline Région métropolitaine & 106 & $49,8 \%$ \\
\hline Non métropolitain & 107 & $50,2 \%$ \\
\hline \multicolumn{3}{|l|}{ Ethnie $(n=92)$} \\
\hline Caucasien & 31 & $33,7 \%$ \\
\hline Non caucasien & 61 & $66,3 \%$ \\
\hline
\end{tabular}


Tableau 1 : Statistiques descriptives des cas de syphilis infectieuse chez les femmess (suite)

\begin{tabular}{|l|r|r|}
\multicolumn{1}{|c|}{ Variables } & \multicolumn{1}{c|}{$n$} & $\%$ \\
\hline Stades infectieux $(\mathrm{n}=\mathbf{2 2 6})$ & 22 & $9,7 \%$ \\
\hline Principale & 49 & $21,7 \%$ \\
\hline Secondaire & 155 & $68,6 \%$ \\
\hline Latente précoce & \multicolumn{2}{|c|}{} \\
\hline Grossesse $(\mathrm{n}=116)$ & 24 & $20,7 \%$ \\
\hline Oui & 92 & $79,3 \%$ \\
\hline Non & &
\end{tabular}

Établissement du diagnostic $(n=148)$

\begin{tabular}{|l|r|r|}
\hline Communauté & 71 & $48,0 \%$ \\
\hline Hôpital & 28 & $18,9 \%$ \\
\hline Sensibilisation & 49 & $33,1 \%$ \\
\hline
\end{tabular}

Raison des tests $(n=217)$

\begin{tabular}{|l|r|r|}
\hline Contact avec une ITS & 34 & $15,7 \%$ \\
\hline Examen médical d'immigration & 5 & $2,3 \%$ \\
\hline Incident & 22 & $10,1 \%$ \\
\hline Prénatal ou à l'accouchement & 23 & $10,6 \%$ \\
\hline Examen de routine & 60 & $27,7 \%$ \\
\hline Symptomatique & 73 & $33,6 \%$
\end{tabular}

ITS récentes $(n=198)$

\begin{tabular}{|l|r|r|}
\hline Oui & 85 & $42,9 \%$ \\
\hline Non & 113 & $57,1 \%$ \\
\hline Séropositif $(\mathrm{VIH})(n=198)$ &
\end{tabular}

Séropositif $(\mathrm{VIH})(\mathrm{n}=198)$

\begin{tabular}{|l|r|r|}
\hline Oui & 8 & $4,0 \%$ \\
\hline Non & 190 & $96,0 \%$ \\
\hline
\end{tabular}

Logement $(n=97)$

\begin{tabular}{|l|r|r|}
\hline Stable & 28 & $28,9 \%$ \\
\hline Instable & 69 & $71,1 \%$ \\
\hline Aucune adresse fixe & 43 & $44,3 \%$ \\
\hline Chambre individuelle/hôtel & 15 & $15,5 \%$ \\
\hline Modulaire/subventionné & 7 & $7,2 \%$ \\
\hline Refuge & 4 & $4,1 \%$ \\
\hline
\end{tabular}

Vie dans la rue $(n=89)$

\begin{tabular}{|l|r|r|}
\hline Oui & 64 & $71,9 \%$ \\
\hline Non & 25 & $28,1 \%$ \\
\hline
\end{tabular}

Rapports sexuels transactionnels $(n=42)$

\begin{tabular}{|l|r|r|}
\hline Oui & 26 & $61,9 \%$ \\
\hline Non & 16 & $38,1 \%$ \\
\hline
\end{tabular}

Consommation de substances $(n=157)$

\begin{tabular}{|l|r|r|}
\hline Non & 50 & $31,9 \%$ \\
\hline Oui & 107 & $68,2 \%$ \\
\hline Alcool & 10 & $6,4 \%$ \\
\hline Stimulants & 17 & $10,8 \%$ \\
\hline Opioïdes & 3 & $1,9 \%$ \\
\hline
\end{tabular}

Tableau 1 : Statistiques descriptives des cas de syphilis infectieuse chez les femmess (suite)

\begin{tabular}{|l|r|r|}
\multicolumn{1}{|c|}{ Variables } & \multicolumn{1}{c|}{$n$} & $\%$ \\
\hline Consommation de substances $(\mathrm{n}=157)$ (suite) & 1 & $0,6 \%$ \\
\hline \multicolumn{1}{|c|}{ Benzodiazépines } & 76 & $48,4 \%$ \\
\hline Polysubstance & 73 & $83,9 \%$ \\
\hline Maladie mentale $(\mathrm{n}=\mathbf{8 7})$ & 14 & $16,1 \%$ \\
\hline Oui & \multicolumn{2}{|c|}{} \\
\hline Non &
\end{tabular}

Aide au revenu $(n=59)$

\begin{tabular}{|l|r|r|}
\hline Oui & 39 & $66,1 \%$ \\
\hline Non & 20 & $33,9 \%$ \\
\hline
\end{tabular}

Historique de l'incarcération $(n=20)$

\begin{tabular}{|l|r|r}
\hline Oui & 17 & $85,0 \%$ \\
\hline Non & 3 & $15,0 \%$ \\
\hline
\end{tabular}

Genre des partenaires $(n=199)$

\begin{tabular}{|l|r|r|}
\hline Femme seulement & 2 & $1,0 \%$ \\
\hline Homme seulement & 191 & $96,0 \%$ \\
\hline Homme et femme & 6 & $3,0 \%$ \\
\hline Nombre de partenaires $(\mathbf{n}=188)$ &
\end{tabular}

\begin{tabular}{|l|r|r|}
\hline Moyenne & 2,6 & s.o. \\
\hline 0 & 8 & $4,3 \%$ \\
\hline 1 & 94 & $50,0 \%$ \\
\hline 2 à 5 & 75 & $39,9 \%$ \\
\hline 6 ou plus & 11 & $5,8 \%$ \\
\hline
\end{tabular}

Notification des partenaires complétée $(n=217)$

\begin{tabular}{|l|r|r|}
\hline Oui & 146 & $67,3 \%$ \\
\hline Non & 71 & $32,7 \%$ \\
\hline Connecté au fournisseur de soins primaires $(n=220)$ \\
\hline Oui & 176 & $80,0 \%$ \\
\hline Non & 44 & $20,0 \%$ \\
\hline
\end{tabular}

Abréviations : ITS, infection transmissible sexuellement; s.o., sans objet; VIH, virus de

l'immunodéficience humaine

a Données du 13 mars au 31 décembre 2018

Le cadre de diagnostic le plus courant était la communauté ( $n=71 / 148,48,0 \%$ ), suivie par la sensibilisation

$(n=49 / 148,33,1 \%)$ et l'hôpital $(28 / 148,18,9 \%)$. La raison la plus courante pour les tests était la présentation de symptômes correspondant à la syphilis $(n=73 / 217,33,6 \%$ ), suivie d'un dépistage de routine $(n=60 / 217,27,7 \%)$ et en raison de la notification d'exposition à une ITS ( $n=34 / 217,15,7 \%)$. Le dépistage prénatal/à l'accouchement de la syphilis était la raison du diagnostic dans environ $10 \%$ des cas $(n=23 / 217)$. Huit cas ont été co-infectés par le VIH, ce qui représente $4,0 \%$ des 198 cas avec un statut de VIH connu. En moyenne, chaque cas comptait 2,6 partenaires précédents. La plupart des cas ont complété le processus de notification des partenaires $(n=146 / 217,67,3 \%)$ et ont été reliés à un prestataire de soins primaires $(n=176 / 220,80,0 \%)$ (tableau 1$)$. 
Parmi les cas où des conditions concomitantes ont été déclarées, $42,9 \%$ ( $n=85 / 198)$ avaient une ITS récente et la majorité avaient connu une instabilité du logement ( $n=69 / 97$, $71,1 \%)$, la vie dans la rue $(n=64 / 89,71,9 \%)$, des rapports sexuels transactionnels ( $n=26 / 42,6,9 \%$ ), la consommation de substances $(n=107 / 157,68,2 \%$ ), la maladie mentale ( $n=73 / 87,83,9 \%$ ) et l'aide au revenu ( $n=39 / 59,66,1 \%)$. De plus, 17 cas ont déclaré un historique d'incarcération, ce qui représentait $85,0 \%(n=17 / 20)$ des personnes ayant des antécédents connus d'incarcération, ou $7,5 \%(n=17 / 226)$ de tous les cas (tableau 1).

Plus de la moitié des cas se trouvaient dans les deux quintiles les plus bas pour la vulnérabilité situationnelle et l'instabilité résidentielle $(52,8 \%$ et $60,3 \%$, respectivement), de I'Indice canadien de défavorisation multiple de Statistique Canada. Les quintiles de composition de la dépendance ethnoculturelle et économique étaient répartis de façon plus égale (figure 1).

Figure 1 : Indice canadien de défavorisation multiple de Statistique Canada $(n=212)^{\text {a }}$

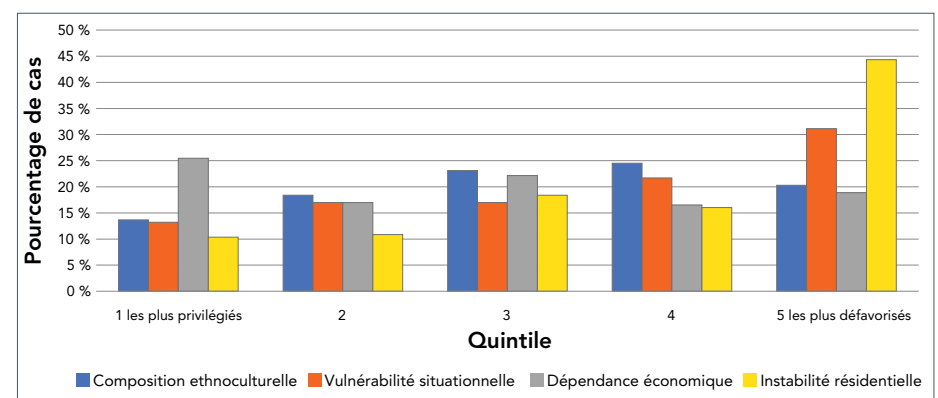

${ }^{a}$ Ces données au niveau de la population sont fondées sur le recensement canadien de 2016, les définitions de chaque indice et des facteurs considérés pour classer le code postal dans les quintiles de défavorisations-privilèges sont disponibles auprès de $:$ https://www150.statcan.gc.ca/ n1/pub/45-20-0001/452000012019002-fra.htm

Il y a eu 24 cas de syphilis infectieuse maternelle, ce qui représente $20,7 \%$ des 116 cas de grossesse connue. L'âge moyen était de 30 ans (intervalle de 23 à 41 ans). Près de la moitié ( $n=11 / 23,47,8 \%$ ) résidaient dans une région métropolitaine. Plus de $90 \%$ ont été diagnostiqués au stade précoce latent de l'infection (tableau 1). En ce qui concerne les conditions concomitantes, de nombreux cas maternels de syphilis ont eu une ITS récente ( $n=8 / 21,38,1 \%$ ) et ont connu une instabilité du logement ( $n=7 / 17,41,2 \%$ ), la vie dans la rue $(n=7 / 12,58,3 \%)$, la consommation de substances ( $n=10 / 17,58,8 \%$ ) et les maladies mentales $n=6 / 9,66,7 \%$ ) (tableau A1 de l'annexe).

Notre analyse sur les quatre sous-groupes a révélé que les cas de logement instable étaient beaucoup plus susceptibles d'avoir des expériences de diagnostic d'une ITS récente, de vivre dans la rue, de rapports sexuels transactionnels, de consommation de substances, de maladie mentale, d'aide au revenu et de ne pas avoir complété la notification au partenaire $(p<0,01$ pour tous). Les cas qui utilisaient des substances étaient beaucoup plus susceptibles d'avoir des expériences de diagnostic d'une
ITS récente, d'un logement instable, d'une vie dans la rue, de rapports sexuels transactionnels, d'une maladie mentale et d'une aide au revenu ( $p<0,01$ pour tous). Les cas diagnostiqués de maladie mentale étaient beaucoup plus susceptibles $d$ 'avoir des antécédents d'instabilité du logement, d'une vie dans la rue et de consommation de substances ( $p<0,01$ pour tous). Enfin, les cas qui ont eu une ITS récente étaient beaucoup plus susceptibles de résider dans une région métropolitaine et d'avoir vécu des antécédents de logement instable, d'une vie dans la rue et de consommation de substances ( $p<0,01$ pour tous) (tableau 2).

\section{Tableau 2 : Analyses de sous-groupes}

\begin{tabular}{|c|c|c|c|c|}
\hline \multirow[b]{2}{*}{ Variables } & \multicolumn{4}{|c|}{ Sous-groupes } \\
\hline & $\begin{array}{c}\text { Instabilité } \\
\text { du } \\
\text { logement } \\
(n=97)\end{array}$ & $\begin{array}{l}\text { Consommation } \\
\text { de substances } \\
(n=157)\end{array}$ & $\begin{array}{l}\text { Maladie } \\
\text { mentale } \\
(n=87)\end{array}$ & $\begin{array}{c}\text { ITS } \\
\text { récentes } \\
(n=198)\end{array}$ \\
\hline Urbanité & n.s. & n.s. & n.s. & 0,0123 \\
\hline ITS récentes & 0,0003 & 0,0025 & n.s. & s.o. \\
\hline $\begin{array}{l}\text { Instabilité du } \\
\text { logement }\end{array}$ & s.o. & $<0,0001$ & 0,0006 & 0,0003 \\
\hline Vie dans la rue & $<0,0001$ & $<0,0001$ & 0,0005 & 0,0062 \\
\hline $\begin{array}{l}\text { Sexe } \\
\text { transactionnel }\end{array}$ & $<0,0001$ & $<0,0001$ & n.s. & n.s. \\
\hline $\begin{array}{l}\text { Consommation } \\
\text { de substances }\end{array}$ & $<0,0001$ & s.o. & 0,0003 & 0,0025 \\
\hline Maladie mentale & 0,0006 & 0,0003 & s.o. & n.s. \\
\hline Aide au revenu & 0,0028 & 0,0003 & n.s. & n.s. \\
\hline $\begin{array}{l}\text { Notification du } \\
\text { partenaire non } \\
\text { complétée }\end{array}$ & 0,0082 & n.s. & n.s. & n.s. \\
\hline
\end{tabular}

Abréviations : ITS, infection transmissible sexuellement; n.s., non significatif; s.o., sans objet

\section{Discussion}

Nous avons décrit tous les cas de syphilis infectieuse chez les femmes diagnostiqués en Colombie-Britannique entre mars 2018 et décembre 2021 afin de mieux comprendre les facteurs de I'infection par la syphilis et les possibilités d'optimiser les soins pour les femmes touchées par la syphilis. Nous avons constaté qu'une proportion élevée des cas de syphilis chez les femmes en Colombie-Britannique ont déclaré une instabilité du logement, une vie dans la rue, une consommation de substances, une maladie mentale et une aide au revenu. Notre analyse de chacun des quatre sous-groupes a révélé des liens étroits entre ces conditions.

Il y a peu d'études axées sur la syphilis infectieuse féminine à l'échelle mondiale. Des études récentes du Manitoba, au Canada, ont révélé une prévalence de conditions concomitantes tout aussi élevée $(13,14)$. Une étude descriptive de la syphilis féminine de 2003 à 2015 a révélé que comparativement aux cas de syphilis chez les hommes, les cas de femmes étaient plus 
susceptibles de déclarer une co-infection avec la chlamydia et la consommation d'alcool (13). De plus, la proportion de cas hétérosexuels de syphilis signalant une co-infection avec la chlamydia, l'instabilité du logement et la consommation de substances était plus élevée entre 2015 et 2018 comparativement à la période de 2011 à 2014, ce qui a coïncidé avec une diminution du ratio hommes-femmes au Manitoba (14). Les auteurs ont conclu qu'il y avait deux épidémies simultanées de syphilis : I'une chez les gbHARSAH et l'autre chez la population hétérosexuelle, cette dernière étant plus difficile à contrôler (14).

Nous avons également constaté qu'une proportion élevée de cas de syphilis infectieuse maternelle présentaient une ITS récente; une conclusion semblable à celle rapportée dans une étude de cas nationale américaine de 2012 à 2016 (15). Notre analyse des cas maternels a révélé une proportion élevée d'une vie dans la rue, de la consommation de substances, de la maladie mentale et de l'aide au revenu, ce qui est conforme à un examen récent de l'Agence de la santé publique du Canada (16). Ces facteurs peuvent également contribuer à des expériences de discrimination et avoir une incidence sur l'accès aux soins prénatals qui ont une incidence sur la santé et le bien-être globaux de la mère et du bébé (16).

La forte association entre les déterminants socio-économiques, les conditions concomitantes et l'infection à la syphilis appuie la nécessité d'une approche fondée sur la syndémie pour répondre à l'épidémie actuelle de syphilis (6,7,17-20). Cela comprend la reconnaissance et la déconstruction des obstacles structurels, comme ceux pour un logement abordable et stable (16). Notre étude a révélé une surreprésentation des femmes atteintes de syphilis dans les quintiles d'instabilité résidentielle les plus défavorisés, mettant l'accent sur la marginalisation des femmes atteintes de la syphilis en Colombie-Britannique.

L'incarcération est également un facteur clé qui se croise et peut exacerber les ITSS. Deux études canadiennes récentes ont révélé que la population carcérale était plus mobile et présentait des taux élevés d'antécédents de consommation de substances, de maladie mentale, de rapports sexuels transactionnels et d'instabilité financière et de logement $(21,22)$. Ces conditions concomitantes se renforcent mutuellement et compliquent le diagnostic et les soins de suivi liés aux ITSS, en raison de la stigmatisation et du manque de lien avec un fournisseur de soins cohérent, ce qui contribue finalement à des résultats de santé précaires $(21,22)$. Ainsi, les partenariats avec les établissements correctionnels fédéraux et provinciaux peuvent aider à soutenir les personnes infectées par la syphilis. Bien que la plupart des établissements correctionnels aient des programmes de dépistage des ITSS, ces programmes varient grandement en ce qui concerne les maladies dépistées, l'approche à l'égard des tests (i.e. l'acceptation volontaire ou l'exclusion) et l'exigence d'être symptomatiques pour être testées pour les ITSS (23-25). Notre étude s'ajoute à la documentation existante qui appuie la mise en œuvre d'un dépistage universel des ITSS axé sur le client dans tous les milieux correctionnels $(23,25)$.

Notre étude peut offrir un aperçu des possibilités d'améliorer les soins de syphilis. Nous avons constaté que près de la moitié des diagnostics se trouvaient dans le milieu communautaire, ce qui pourrait être un domaine d'investissement pour mieux traiter à la fois la syphilis et les autres épidémies croissantes, comme l'instabilité du logement, la consommation de substances et la maladie mentale, par la co-implantation de soutiens supplémentaires comme les services de travailleurs sociaux et de counseling (19). En outre, la collaboration avec les services d'urgence, les centres de soins d'urgence et les établissements correctionnels en vue d'élaborer des normes et des ressources adaptées pour ces établissements peut aider à identifier les cas de syphilis et à améliorer les liens avec les équipes de soins et de sensibilisation en santé publique. Bien que ce ne soit pas le cadre dans lequel la majorité des cas de notre étude ont été diagnostiqués, les services d'urgence sont fréquemment utilisés par les populations qui n'ont pas de fournisseur de soins primaires ou qui sont marginalisées par l'instabilité financière, le manque de logements et autres systèmes de soutien (16).

\section{Limites}

Cette étude comporte plusieurs limites. Premièrement, I'examen des graphiques ne saisissait pas l'ensemble des antécédents médicaux et sociaux de chaque cas. Par conséquent, la présence ou l'absence de facteurs de risque et de conditions concurrentes n'était pas connue dans tous les cas et certaines variables avaient des échantillons de plus petite taille. Par exemple, il y avait peu de données non spécifiques documentées dans les graphiques des clients concernant l'origine ethnique des cas. Une meilleure compréhension des expériences différentielles des groupes ethniques a déjà été identifiée comme une priorité pour d'autres travaux en Colombie-Britannique $(26,27)$. Deuxièmement, seuls les cas de syphilis infectieuse diagnostiqués pouvaient être analysés; par conséquent, certaines populations qui n'ont pas accès, qui ne sont pas sensibilisées ou qui n'ont pas d'organisme pour subir des tests de dépistage de syphilis, auraient été sous-représentées dans cette étude. Enfin, cette étude était de nature descriptive et ne démontre pas de causalité entre les facteurs d'infection et le diagnostic de syphilis. Cependant, nos constatations sont conformes à la documentation et renforcent les données probantes sur l'augmentation des services pour traiter les conditions socioéconomiques et concurrentes dans la réponse à l'épidémie de syphilis.

\section{Conclusion}

Dans l'ensemble, cette étude a caractérisé tous les cas de syphilis infectieuse chez les femmes diagnostiquées en Colombie-Britannique sur une période de près de trois ans. Nous avons constaté que sur les 226 cas de syphilis infectieuse chez les femmes, une proportion importante présentait des conditions concomitantes, y compris l'instabilité du logement, la consommation de substances et les maladies mentales. À notre connaissance, il s'agit de la plus importante analyse 
descriptive de la syphilis infectieuse chez les femmes au Canada. De plus, l'utilisation de systèmes d'information clinique provinciaux complets a permis de mieux comprendre le contexte socioéconomique des cas de syphilis infectieuse. Les résultats de notre étude peuvent aider à suggérer des interventions visant à promouvoir des conditions favorables pour prévenir et optimiser les soins des femmes affectées par la syphilis, tel que l'intégration des mesures de soutien en matière de maladie mentale et de toxicomanie aux soins des ITS.

\section{Déclaration des auteurs}

K. W. - Conceptualisation, méthodologie, enquête, traitement des données, rédaction de la version originale, révision et édition L. B. - Conceptualisation, méthodologie, enquête, révision et édition

S. R. - Révision et édition

I. P. - Enquête, révision et édition

V. R. - Conceptualisation, méthodologie, traitement des données, révision et édition

A. Y. - Traitement des données, révision et édition

A. P. - Révision et édition

G. O. - Révision et édition

T. G. - Conceptualisation, révision et édition

J. W. - Conceptualisation, méthodologie, rédaction d'une

ébauche originale, révision et édition, administration du projet

Le contenu de l'article et les points de vue qui y sont exprimés n'engagent que les auteurs et ne correspondent pas nécessairement à ceux du gouvernement du Canada.

\section{Intérêts concurrents}

Les auteurs n'ont aucun intérêt concurrent à déclarer.

\section{Remerciements}

Les auteurs aimeraient remercier E. Wong pour sa contribution à cette étude. Nous aimerions également reconnaître le Laboratoire de santé publique du BCCDC et l'équipe clinique de lutte contre la syphilis pour leur rôle continu dans la prise en charge et la défense des personnes touchées par la syphilis.

\section{Financement}

Aucun financement externe n'a été reçu pour ce projet.

\section{Références}

1. Agence de la santé publique du Canada. Syphilis infectieuse et syphilis congénitale au Canada, 2020 (infographie). Ottawa (ON) : ASPC; 2021 (accédé 2021-08-03). https:// www.canada.ca/fr/sante-publique/services/publications/ maladies-et-affections/syphilis-infectieuse-syphiliscongenitale-canada-2020.html

2. British Columbia Centre for Disease Control. STI Reports. Vancouver (BC): BCCDC; 2021 (accédé 2021-06-22). http:// www.bccdc.ca/health-professionals/data-reports/sti-reports

3. Ablona A, Chang HJ, Grace D, Worthington C, Wong J, Ogilvie G, Gilbert M. Sex in the Time of COVID-19: Preliminary results from a survey conducted on July 21 to August 4, 2020. Vancouver (BC): DISHI; 2020 (accédé 2021-11-03). https://dishiresearch.ca/resource/sex-inthe-time-of-covid-19-preliminary-results-from-a-surveyconducted-on-july-21-to-august-4-2020/

4. British Columbia Centre for Disease Control. British Columbia Syphilis Indicators 2021 Q3. Vancouver (BC): BCCDC; 2021 (accédé 2021-12-27). http://www.bccdc. ca/resource-gallery/Documents/Statistics\%20and\%20 Research/Statistics\%20and\%20Reports/STI/BC_syphilis_ indicators_2021Q3_FINAL.pdf

5. British Columbia Centre for Disease Control. Syphilis Action Plan. Vancouver (BC): BCCDC; 2016 (accédé 2021-05-27). http://www.bccdc.ca/resource-gallery/Documents/ Statistics\%20and\%20Research/Statistics\%20and\%20Reports/ STI/Syphilis\%20Action\%20Plan.pdf

6. Agence de la santé publique du Canada. Réduction des répercussions sur la santé des infections transmissibles sexuellement et par le sang au Canada d'ici 2030 : un cadre d'action pancanadien sur les ITSS. Ottawa (ON) : ASPC; 2018 (accédé 2021-08-03). https://www.canada.ca/ $\mathrm{fr} / \mathrm{sante}$-publique/services/maladies-infectieuses/santesexuelle-infections-transmissibles-sexuellement/rapportspublications/infections-transmissibles-sexuellement-sangcadre-action.html

7. Wong J, Ryan V, Bharmal A, Morshed M, Ford G, Mooder K, Giesbrecht E, Pederson A, Grennan T, Gilbert M. O06.6 Refreshing the British Columbia Syphilis Control Strategy: Thematic Analysis of a Multi-Stakeholder Consultation Process. Sex Transm Infect 2021;97 Suppl 1:A29-30. DOI

8. Singer M. Introduction to Syndemics: A Critical System Approach to Public and Community Health. San Francisco, CA: Jossey-Bass, 2009. ISBN: 978-0-470-47203-3

British Columbia Centre for Disease Control. Syphilis. Vancouver (BC): BCCDC; 2021 (accédé 2021-06-11). http://www.bccdc.ca/health-professionals/clinical-resources/ case-definitions/syphilis 
9. Vancouver Coastal Health. CareConnect. BC: VCH; 2020 (accédé 2021-09-03). http://www.vch.ca/for-healthprofessionals/resources-updates/careconnect

10. Province of British Columbia. Health Boundaries. Victoria (BC): Government of British Columbia; 2021 (accédé 2021-08-11). https://www2.gov.bc.ca/gov/content/ data/geographic-data-services/land-use/administrativeboundaries/health-boundaries

11. Statistique Canada. L'Indice canadien de défavorisation multiple : Ensemble de données et guide de I'utilisateur. Ottawa (ON) : StatCan; 2019 (accédé 2021-06-11). https://www150.statcan.gc.ca/n1/fr/catalogue/45200001

12. Shaw SY, Ross C, Nowicki DL, Marshall S, Stephen S, Davies C, Riddell J, Bailey K, Elliott LJ, Reimer JN, Plourde PJ. Infectious syphilis in women: what's old is new again? Int J STD AIDS 2017;28(1):77-87. DOI PubMed

13. Shaw S, Lapple A, Reimer J, Ross C, Nowicki D, Elliott L, Ploudre P. P768 The evolution of an infectious syphilis epidemic in a Canadian urban setting (Poster PS02). Sex Transm Infect 2019;95 Suppl 1:A330. DOI

14. Trivedi S, Williams C, Torrone E, Kidd S. National Trends and Reported Risk Factors Among Pregnant Women With Syphilis in the United States, 2012-2016. Obstet Gynecol 2019;133(1):27-32. DOI PubMed

15. Agence de la santé publique du Canada. La Syphilis au Canada : Rapport technique sur les tendances épidémiologiques, les determinants et interventions. Ottawa (ON) : ASPC; 2020 (accédé 2021-06-11). https://epe.lac-bac. gc.ca/100/201/301/weekly_acquisitions_list-ef/2021/21-12/ publications.gc.ca/collections/collection_2021/aspc-phac/ HP40-267-2020-fra.pdf

16. Ferlatte O, Salway T, Samji H, Dove N, Gesink D, Gilbert M, Oliffe JL, Grennan T, Wong J. An Application of Syndemic Theory to Identify Drivers of the Syphilis Epidemic Among Gay, Bisexual, and Other Men Who Have Sex With Men. Sex Transm Dis 2018;45(3):163-8. DOI PubMed

17. Kidd SE, Grey JA, Torrone EA, Weinstock HS. Increased Methamphetamine, Injection Drug, and Heroin Use Among Women and Heterosexual Men with Primary and Secondary Syphilis - United States, 2013-2017. MMWR Morb Mortal Wkly Rep 2019;68(6):144-8. DOI PubMed

18. Salway T, Ferlatte $O$, Shoveller J, Purdie A, Grennan T, Tan DH, Consolacion T, Rich AJ, Dove N, Samji H, Scott K, Blackwell E, Mirau D, Holgerson N, Wong J, Gilbert M. The Need and Desire for Mental Health and Substance Use-Related Services Among Clients of Publicly Funded Sexually Transmitted Infection Clinics in Vancouver, Canada. J Public Health Manag Pract 2019;25(3):E1-10. DOI PubMed
19. Williams SP, Bryant KL. Sexually Transmitted Infection Prevalence among Homeless Adults in the United States: A Systematic Literature Review. Sex Transm Dis 2018;45(7):494-504. DOl PubMed

20. National Collaborating Centre for Infectious Diseases. Incarceration and sexually transmitted and bloodborne diseases. Winnipeg (MB): NCCID; 2021 (accédé 2021-12-12). https://nccid.ca/publications/incarceration-and-stbbis/

21. Taylor S, Haworth-Brockman M, Keynan Y. Slipping through: mobility's influence on infectious disease risks for justice-involved women in Canada. Health Justice 2021;9(1):35. DOl PubMed

22. Bartlett SR, Buxton J, Palayew A, Picchio CA, Janjua NZ, Kronfli N. Hepatitis C Virus Prevalence, Screening, and Treatment Among People Who Are Incarcerated in Canada: Leaving No One Behind in the Direct-Acting Antiviral Era. Clin Liver Dis (Hoboken) 2021;17(2):75-80. DOI PubMed

23. Reekie A, Gratrix J, Ahmed R, Smyczek P. P138 Evaluating Opt-Out STI Testing at Admission within a Short-Term Correctional Facility Located in Alberta, Canada. Sex Transm Infect 2021;97:A95. DOI

24. Kronfli N, Dussault C, Bartlett S, Fuchs D, Kaita K, Harland K, Martin B, Whitten-Nagle C, Cox J. Disparities in hepatitis $C$ care across Canadian provincial prisons: Implications for hepatitis C micro-elimination. Can Liver J 2021;4(3):292-310. https://canlivj.utpjournals.press/doi/abs/10.3138/ canlivj-2020-0035

25. Turpel-Lafond ME. In Plain Sight: Addressing Indigenous-specific Racism and Discrimination in B.C. Health Care. Government of British Columbia; 2020 (accédé 2021-12-16). https://engage.gov.bc.ca/app/uploads/ sites/613/2020/11/In-Plain-Sight-Full-Report.pdf

26. British Columbia's Office of the Human Rights Commissioner. Disaggregated demographic data collection in British Columbia: The grandmother perspective. Vancouver (BC): BCOHR; 2020 (accédé 2021-12-16). https://bchumanrights. ca/wp-content/uploads/BCOHRC_Sept2020_DisaggregatedData-Report_FINAL.pdf 


\section{Annexe Tableau A1 : Statistiques descriptives de la syphilis maternelle $(n=24)$}

\begin{tabular}{|c|c|c|}
\hline Variables & $\mathbf{n}$ & $\%$ \\
\hline \multicolumn{3}{|l|}{ Âge (années) $(n=24)$} \\
\hline Moyenne & 30,6 & s.o. \\
\hline Médiane & 30,0 & s.o. \\
\hline Min. & 23,3 & s.o. \\
\hline Max. & 41,7 & s.o. \\
\hline \multicolumn{3}{|l|}{ Année - total $(n=24)$} \\
\hline $2018^{a}$ & 2 & $8,3 \%$ \\
\hline 2019 & 7 & $29,2 \%$ \\
\hline 2020 & 15 & $62,5 \%$ \\
\hline \multicolumn{3}{|c|}{ Année - nombre moyen de cas par mois $(n=24)$} \\
\hline $2018^{a}$ & 0,2 & s.o. \\
\hline 2019 & 0,6 & s.o. \\
\hline 2020 & 1,3 & s.o. \\
\hline \multicolumn{3}{|l|}{ Autorités sanitaires $(n=23)$} \\
\hline Nord & 1 & $4,3 \%$ \\
\hline Intérieur & 4 & $17,4 \%$ \\
\hline Île de Vancouver & 4 & $17,4 \%$ \\
\hline Fraser & 7 & $30,4 \%$ \\
\hline Vancouver Coastal & 7 & $30,4 \%$ \\
\hline \multicolumn{3}{|l|}{ Urbanité $(n=23)$} \\
\hline Région métropolitaine & 11 & $47,8 \%$ \\
\hline Non métropolitain & 12 & $52,2 \%$ \\
\hline \multicolumn{3}{|l|}{ Ethnie $(n=11)$} \\
\hline Caucasien & 5 & $45,5 \%$ \\
\hline Non caucasien & 6 & $54,5 \%$ \\
\hline \multicolumn{3}{|l|}{ Stades infectieux ( $n=24$ ) } \\
\hline Principale & 0 & $0 \%$ \\
\hline Secondaire & 1 & $4,2 \%$ \\
\hline Latente précoce & 23 & $95,8 \%$ \\
\hline \multicolumn{3}{|c|}{ Configuration du diagnostic $(n=17)$} \\
\hline Communauté & 13 & $76,5 \%$ \\
\hline Hôpital & 2 & $11,8 \%$ \\
\hline Sensibilisation & 2 & $11,8 \%$ \\
\hline \multicolumn{3}{|l|}{ Raison des tests $(n=24)$} \\
\hline Contact avec une ITS & 1 & $4,2 \%$ \\
\hline Prénatal ou à l'accouchement & 21 & $87,5 \%$ \\
\hline Examen de routine & 2 & $8,3 \%$ \\
\hline Symptomatique & 0 & $0 \%$ \\
\hline \multicolumn{3}{|l|}{ ITS récentes $(n=21)$} \\
\hline Oui & 8 & $38,1 \%$ \\
\hline Non & 13 & $61,9 \%$ \\
\hline \multicolumn{3}{|l|}{ Séropositif $(\mathrm{VIH})(\mathrm{n}=21)$} \\
\hline Oui & 0 & $0 \%$ \\
\hline Non & 21 & $100 \%$ \\
\hline \multicolumn{3}{|l|}{ Logement $(n=17)$} \\
\hline Stable & 10 & $58,8 \%$ \\
\hline Pas stable & 7 & $41,2 \%$ \\
\hline
\end{tabular}

\begin{tabular}{|l|r|r|}
\multicolumn{1}{|c|}{ Variables } & n & \multicolumn{1}{c|}{$\%$} \\
\hline Logement $(\mathbf{n}=17)$ (suite) & 5 & $29,4 \%$ \\
\hline Aucune adresse fixe & 2 & $11,8 \%$ \\
\hline Chambre individuelle/hôtel & 0 & $0 \%$ \\
\hline Modulaire/subventionné & 0 & $0 \%$ \\
\hline Refuge & &
\end{tabular}

Vie dans la rue $(n=12)$

\begin{tabular}{|l|r|r|}
\hline Oui & 7 & $58,3 \%$ \\
\hline Non & 5 & $41,7 \%$ \\
\hline Rapports sexuels transactionnels $(\boldsymbol{n}=4)$ & 1 & $25,0 \%$ \\
\hline Oui & 3 & $75,0 \%$ \\
\hline Non & 7 & $41,2 \%$ \\
\hline Consommation de substances $(\boldsymbol{n}=17)$ & 10 & $58,8 \%$ \\
\hline Non & 1 & $5,9 \%$ \\
\hline Oui & 3 & $17,6 \%$ \\
\hline Alcool & 0 & $0 \%$ \\
\hline Stimulants & 0 & $0 \%$ \\
\hline Opiö̈des & 6 & $35,3 \%$ \\
\hline Benzodiazépines & & \\
\hline Polysubstances & &
\end{tabular}

Maladie mentale $(n=9)$

\begin{tabular}{|l|r|r|}
\hline Oui & 6 & $66,7 \%$ \\
\hline Non & 3 & $33,3 \%$ \\
\hline
\end{tabular}

\section{Aide au revenu $(n=4)$}

Oui

\begin{tabular}{l|l|}
\hline 3 & $75,0 \%$ \\
\hline 1 & $25,0 \%$ \\
\hline
\end{tabular}

Historique de l'incarcération $(n=2)$

\begin{tabular}{|l|r|r|}
\hline Oui & 2 & $100 \%$ \\
\hline Non & 0 & $0 \%$ \\
\hline
\end{tabular}

Genre des partenaires $(n=19)$

\begin{tabular}{|l|r|r|}
\hline Femme & 0 & $0 \%$ \\
\hline Homme & 19 & $100 \%$ \\
\hline Homme et femme & 0 & $0 \%$ \\
\hline
\end{tabular}

Nombre de partenaires $(n=21)$

\begin{tabular}{|l|r|r|}
\hline Moyenne & 1,8 & s.o. \\
\hline 1 & 15 & $71,4 \%$ \\
\hline 2 à 5 & 5 & $23,8 \%$ \\
\hline 6 ou plus & 1 & $4,8 \%$ \\
\hline
\end{tabular}

\section{Notification des partenaires complétée $(n=23)$}

\begin{tabular}{|l|r|r|}
\hline Oui & 17 & $73,9 \%$ \\
\hline Non & 6 & $26,1 \%$ \\
\hline
\end{tabular}

Connecté au fournisseur de soins primaires $(n=24)$

\begin{tabular}{|l|r|r|}
\hline Oui & 20 & $83,3 \%$ \\
\hline Non & 4 & $16,7 \%$ \\
\hline
\end{tabular}

Abréviations : ITS, infection transmissible sexuellement; s.o., sans objet; $\mathrm{VIH}$, virus de

l'immunodéficience humaine

a Données du 13 mars au 31 décembre 2018 